\title{
Pedigree construction and disease confirmation: a pilot study in Wales exploring the role of nonclinical personnel
}

\author{
Vanessa Tempest ${ }^{1}$, Rachel Iredale ${ }^{*, 1}$, Jonathon Gray $^{2}$, Liz France ${ }^{2}$, Sally Anstey ${ }^{2}$ \\ and John Steward ${ }^{3}$ \\ ${ }^{1}$ Institute of Medical Genetics, School of Medicine, Cardiff University, Cardiff, UK; ${ }^{2}$ Institute of Medical Genetics, \\ Cardiff, UK; ${ }^{3}$ Welsh Cancer Intelligence and Surveillance Unit (WCISU), Cardiff, UK
}

Pedigree construction and disease confirmation are the means by which reported family histories are translated into a verified clinical tool informing risk assessment and management decisions by clinical genetics staff. In this study, we hypothesised that pedigree generation data processes do not generally require the clinical expertise of genetic counsellors and that they could be successfully transferred to nonclinical data administrators. We made a pragmatic comparison of two processes of pedigree generation by different personnel from 14 consecutive family history questionnaires containing 88 living and decease affected individuals. The pedigrees generated by the genetic counsellor and the data administrator were compared; discrepancies were quantified and their source determined. The information gathered by the data administrator mirrored that of the genetic counsellors in $89 \%$ of cases. Time was saved by permitting direct access to cancer registry and local oncology centre databases. Constructing a pedigree is not always a case of transferring clear-cut data. Decisions need to be made about which cancers to confirm. Notable differences emerged in the number of pieces of information not transferred. Ambiguous information was often interpreted differently, suggesting the need for clinical staff to review pedigrees after their initial plotting by the data administrator. This study demonstrates a good degree of concordance between pedigrees constructed by a nonclinical data administrator and those of experienced genetic counsellors. However, the redirection of all pedigree activity to nonclinical personnel up to the point of risk review is not possible at present. European Journal of Human Genetics (2005) 13, 1063-1070. doi:10.1038/sj.ejhg.5201454; published online 15 June 2005

Keywords: cancer genetics; family history; pedigree; genetic counsellor; data; cancer registers

\section{Introduction}

The recognition of the genetic component of certain common cancers has led to an increase in public demand for information, reassurance and cancer screening, and consequently in significant increases in referrals to clinical

*Correspondence: $\mathrm{Dr} \mathrm{R}$ Iredale, Institute of Medical Genetics, Cardiff University School of Medicine, Heath Park, Cardiff CF14 4XN, UK. Tel: + 44292074 8925; Fax: + 44292074 8927;

E-mail: Rachel.Iredale@cardiffandvale.wales.nhs.uk

Received 17 February 2005; revised 27 April 2005; accepted 18 May 2005; published online 15 June 2005 genetic centres. ${ }^{1,2}$ This increase in referrals requires major additional investment in risk assessment, genetic counselling and testing resources. ${ }^{3,4}$ Traditionally, the increased workload has been met by additional medically or nursing qualified staff. ${ }^{5}$ This pilot study explored the possibility of role substitution of nonclinical personnel for genetic counsellors for pedigree construction and disease confirmation.

The need for a detailed and accurate family history The first step in the provision of cancer genetic services is the collection of an accurate and detailed family history to 
assess whether cancers in the proband's relatives may be due to an inherited susceptibility rather than a sporadic occurrence. ${ }^{6,7}$ Information is required about cancers in maternal and paternal relations, as well as details of unaffected relatives. Upon this data, risk estimates are calculated - average (ie population), moderate or high risk - and decisions taken, such as reassurance, surveillance, genetic testing or prophylactic surgery.

Unfortunately, family histories as reported to genetics services are not always correct, so although time consuming, the confirmation of reported cancers is routinely sought from cancer registries, medical notes and death certificates. The accuracy of reported cancers generally varies according to cancer site and proximity of relationship to the referral. ${ }^{8,9}$ Accuracy is not related to the proband's age or gender, nor to the reason for referral or personal history of cancer. ${ }^{10}$ Ziogas and Anton-Culver ${ }^{11}$ found family history is usually more accurate for firstdegree relatives than for second- and third-degree relatives. Inaccuracies can affect the validity of clinical management decisions and the generalisability of results from screening trials. Douglas et $a l^{12}$ found that management was altered as a result of additional information from disease confirmations in $11 \%$ of the families studied. In Scotland, Brewster et $\mathrm{l}^{13}$ found the information fed back from cancer registries to genetics clinics led to changes in family history, risk categorisation and management in 41, 30 and $23 \%$ of cases, respectively. The success in retrieving medical records declines with increasing record age. ${ }^{14}$ Studies suggest that patient-reported family histories are accurate for breast and colon cancer, and that it may not be necessary to confirm all diagnoses rountinely. ${ }^{9,15}$

Cancer registries are only as good as the data they hold. Ways of collecting data differ across Europe. For example, in Denmark, voluntary notifications are received by cancer registries directly from general practitioners, hospitals or practising specialists. In Italy pilot studies have explored the feasibility of using computer-assisted systems in data collection. ${ }^{16}$ In the Netherlands regional cancer registries use a system of active data collection by trained registration personnel, as do registries in the UK and the US. The quality of cancer registry data has received much attention but very few studies have been published on this issue. ${ }^{15}$

Pedigree construction and disease confirmation are the means by which reported family histories are translated into a verified clinical tool informing risk assessment and management decisions. A pedigree needs to be carefully constructed, drawing on accurate information and employing standardised and consistent nomenclature. It summarises medical information and family relationships in abbreviated notation aiding interpretation of the family history ${ }^{17}$ and is used to facilitate case review between clinicians. ${ }^{18}$ In addition, the pedigree exists as a sociological aid to counselling by serving as a record of family social relationships.

\section{Cancer Genetics Service for Wales}

The Cancer Genetics Service for Wales (CGSW) was established in 1998 following recommendations made by the Calman-Hine ${ }^{19}$ and Harper Reports. ${ }^{20}$ At CGSW family history data are collected through postal family history questionnaires, which request details of cancers in relatives, as well as details of unaffected relatives. Upon return of the questionnaire, information is transferred to a family pedigree and depending on an initial judgement of the level of risk (average, moderate or high), confirmations will be sought in certain cases. As a general rule, cancers are only confirmed for high-risk referrals and for moderate risk if there are incidences of ovarian cancer. These confirmations are sought from: regional cancer registries (most commonly the Welsh Cancer Intelligence and Surveillance Unit (WCISU)); medical records (often from the local oncology centre, Velindre Hospital) and pathology reports. This information is then appended to the pedigree.

CGSW uses the Cyrillic 2.1 pedigree software combined with the MLINK linkage programme. Cyrillic employs the Claus model to calculate the cumulative risks of developing cancer and the numerical risk of carrying a mutation in a particular cancer susceptibility gene. ${ }^{21}$ Currently, at CGSW genetic counsellors are responsible for the transfer of family history questionnaire data to pedigrees and disease confirmations. Other responsibilities include stratifying individuals' risks; establishing a relationship with the proband; and communicating risk information. As demand rises, the genetic counsellor is also increasingly involved in educating patients, other health professionals and trainee colleagues. $^{22,23}$ The significant increase in referrals to CGSW has placed considerable burdens on its genetic counsellors with competing responsibilities. The average number of monthly referrals has increased from 13 in 1998 to 160 in 2004 . Concern over both the timely processing of individuals (whose anxiety may be raised by referral), and with efficient human resource allocation prompted an exploration of the way pedigree generation activity is organised at CGSW.

\section{A new model for pedigree generation}

The study aimed to ascertain whether pedigree construction and disease confirmation are activities that can be transferred to nonclinical administrators, while maintaining the quality of confirmed pedigree generated. It was hypothesised that pedigree generation data processes do not generally require the clinical expertise of trained genetic counsellors. Liberated from the administrative burden of pedigree 'work-up', genetic counsellors could fully direct their knowledge and experience toward risk determination, management and counselling. Additionally, a data administrator would be more likely to have the necessary skills and experience to perform such repetitive data transfer tasks reliably. 
The activities involved in constructing a pedigree are labour intensive and often subject to delays beyond the control of CGSW. Consent forms from family members and requests for notes and confirmations from other clinical establishments are not always promptly returned. Therefore, another strategy for improving efficiency in this pilot study was to provide the data administrators with direct access to the WCISU database and the database at Velindre Hospital - Information System for Clinical Organisations (ISCO), rather than issuing postal requests. A dedicated data administrator with immediate access to cancer registry data could process the information more quickly, greatly facilitating the clinical process. Ultimately, this should reduce the time between referral and communication of risk to the client.

\section{Method}

This study was a pragmatic comparison of two processes of pedigree generation by personnel with differing backgrounds and competencies. The first process was the standard CGSW procedure for 'working up' pedigrees by two Cardiff-based genetic counsellors. Upon receipt of the family history questionnaire, initial Cyrillic pedigrees were plotted and reviewed, whereupon a decision was made as to the necessary cancers to confirm. In certain cases, where information on the questionnaire was ambiguous or scanty, the genetic counsellor contacted the referred individual by phone for clarification. Written consent for the release of information was sought for living affected individuals via the proband. Postal requests for confirmations were made to the appropriate place. Reports were returned by post with the required information or notification that no information was available; the Cyrillic pedigrees were then updated with relevant data.

The second process was conducted by a nonclinical data administrator, (author VT), a researcher with a humanities background. From a copy of the same set of family history questionnaires, Cyrillic pedigrees were plotted. The data administrator noted the questionnaires which would have necessitated contact with the proband to clarify information. Confirmations were conducted in person by the data administrator at WCISU where computer access was provided to WCISU data and to ISCO at Velindre Hospital. Certain genetic counsellor activities could not be duplicated such as: obtaining consent from living affected relatives for access to medical notes; and submission of requests to other cancer registries and hospitals. Although data administrator confirmations could only be obtained from WCISU and Velindre Hospital, this limitation allowed evaluation of the extent to which these resources would meet the information needs of the model. Consent forms for living affected relatives were passed to the data administrator enabling searches on the WCISU and
Velindre databases for these individuals. The data administrator sought confirmations for all deceased affected individuals on the pedigrees (Figure 1).

Pedigrees were plotted from 14 consecutive family history questionnaires. The pedigrees generated by the data administrator and genetic counsellors were compared; discrepancies were quantified and their source determined. Given that the genetic counsellors had confirmed only a selected number of cancers, it was important to make comparisons on a case-by-case basis. A cumulative comparison would be meaningless given that the genetic counsellors had selected the cases according to the relevance of the family history in question. Should the data administrator have confirmed a greater number of cancers overall, this would not ipso facto mean better quality pedigrees as the extra cases may have been ones of low clinical relevance that the counsellors had chosen not to confirm.

Therefore, genetic counsellor activity and data administrator activity were initially compared for the cases for which the genetic counsellors had sought confirmation. When analysing cases where confirmation had been sought, the source of the confirmation was considered, given that the data administrator was limited to WCISU and Velindre Hospital data resources. The second aspect of data administrator activity examined was the cases that the genetic counsellors had decided not to confirm. Other results presented in this paper include differences in the pedigrees constructed by each (the number of individuals plotted; differences in family relationships; differences in clinical/medical information; and differences in other individual details).

\section{Results \\ Confirmations}

The 14 pedigrees contained 88 living and deceased affected individuals. The genetic counsellors decided that confirmations were necessary in 22 cases (25\%). In five of these cases, consent had been requested but not obtained at the time of the study, leaving 17 cases where confirmation had been sought (nine deceased, eight living). Of these, six requests (35\%) were made to WCISU and five (29\%) to Velindre Hospital. Three requests (18\%) were made to other cancer registries and three to other hospitals (18\%). Figure 2 displays a breakdown of this counsellor activity. Fifteen of the 17 requests $(88 \%)$ were successful. The other two requests, (one each to WCISU and the National Cancer Registry Ireland) yielded no information.

For these 17 cases the data administrator was unable to confirm the six cases where the counsellors had requested information from other cancer registries or hospitals. In two cases, notification of consent had not been passed on and the remaining four individuals were treated outside Wales. Among the 11 cases where the genetic counsellor had requested information from WCISU or Velindre, the 


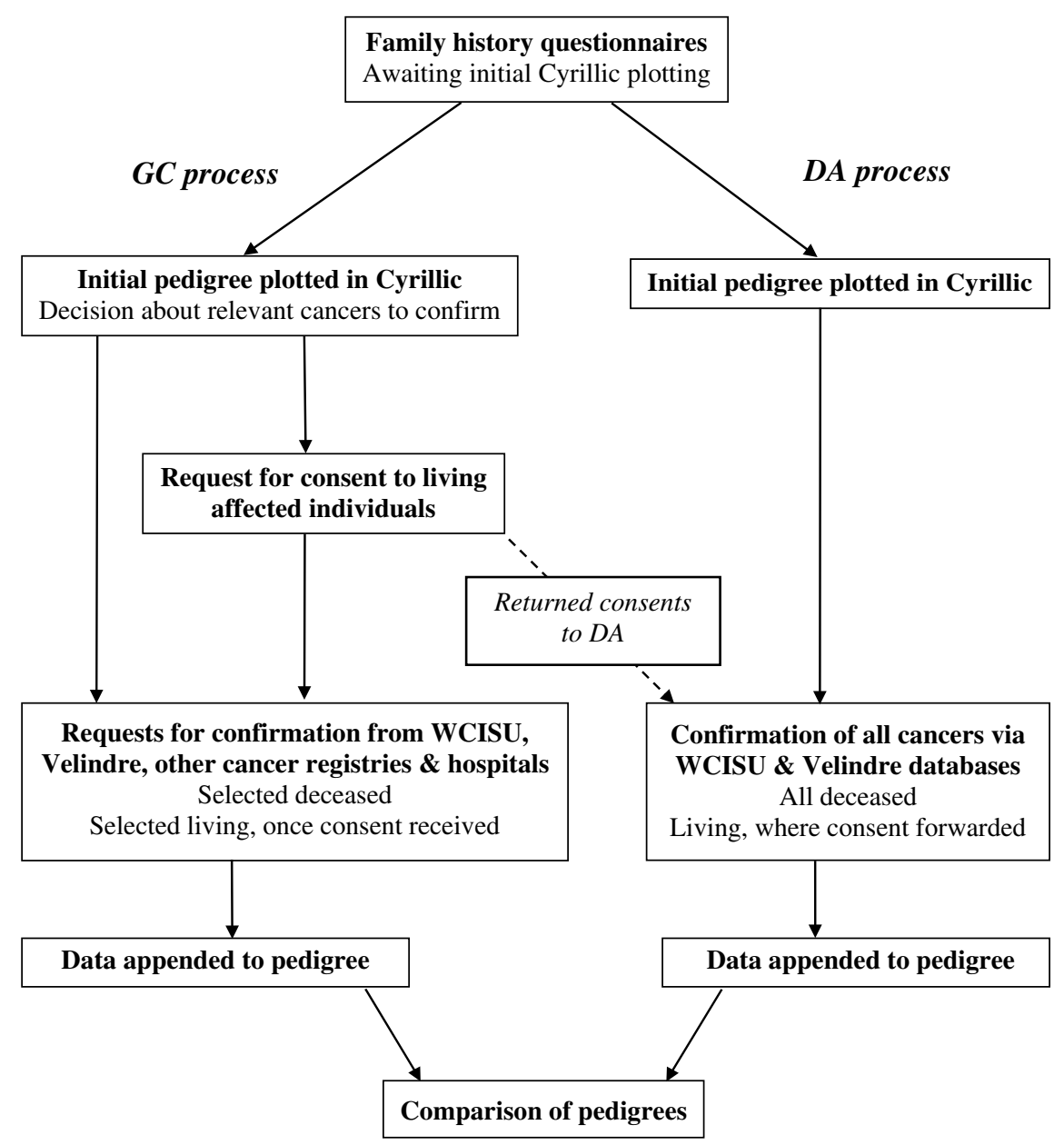

Figure 1 Flow diagram of the pedigree construction and disease confirmation process conducted by the genetic counsellors (GCs) and data administrator (DA).

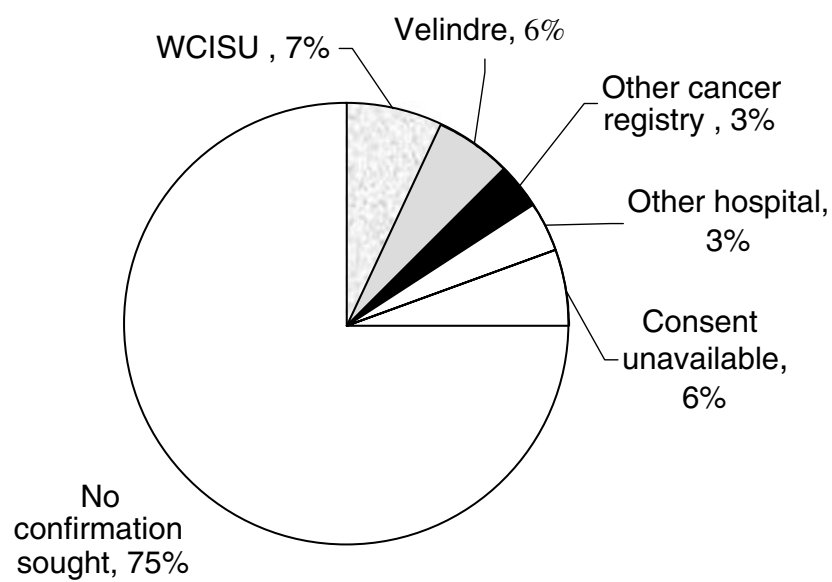

Figure 2 Breakdown of genetic counsellor activity for affected individuals $(n=88)$. data administrator was unable seek confirmations in two cases as notification of consent had not been passed on at the time of the study. Where able (in nine cases), the data administrator data mirrored that retrieved by the genetic counsellor in eight cases (89\%): seven successful confirmations and one unsuccessful confirmation. The other unsuccessful data administrator confirmation had been confirmed by the counsellor via a WCISU request.

The genetic counsellor did not seek confirmations for 66 of the 88 (75\%) affected individuals (See Figure 3). The data administrator did not attempt confirmation in 20 cases; in four of these, information from the family history questionnaire did not suggest confirmations were necessary. The remaining cases were not confirmed as they were living affected relatives from whom no consent had been requested by the genetic counsellor for accessing notes. In the majority of cases for which confirmations were attempted no information was found. Seven cases were confirmed although one only yielded partial information compared to that stated on the family history question- 
naire. The reasons for not locating information included date of diagnosis predating cancer registration, individual treated outside Wales and insufficient detail to perform search.

\section{Pedigree differences}

Table 1 details the differences between the genetic counsellors' and the data administrator's pedigrees.

Individuals plotted The genetic counsellors had additional individuals plotted on four of the 14 pedigrees. This accounted for 28 individuals with, on each pedigree, 2, 3, 6 and in one case 17 extra persons. In all four pedigrees, the

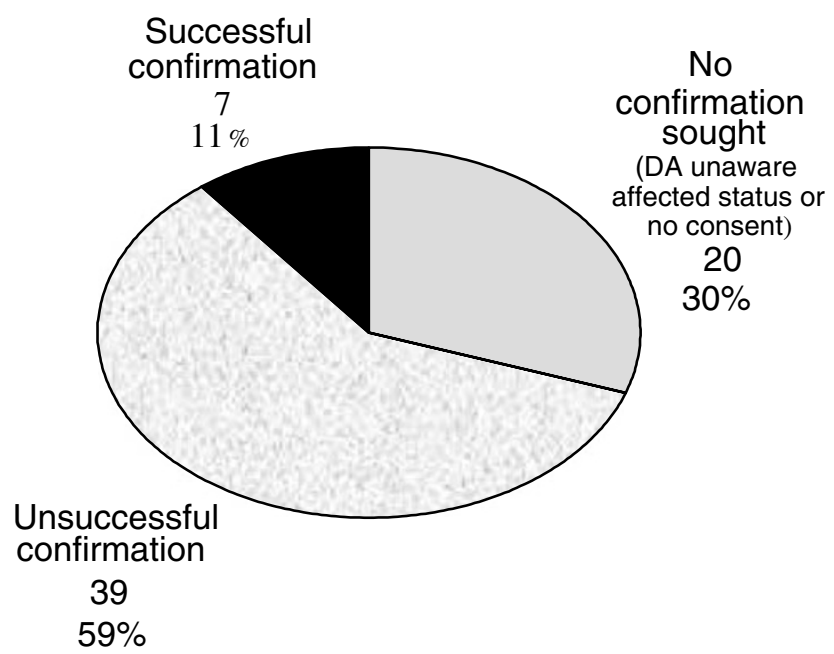

Figure 3 Data administrator confirmations for cases genetic counsellor did not confirm $(n=66)$. genetic counsellors had made a telephone call to the patient to confirm the family history after receipt of the questionnaire and make prerisk assessment. The data administrator had plotted seven extra individuals on four further pedigrees $(+1$ on three pedigrees, +4 on one). On three of these pedigrees the genetic counsellor had not transferred the information from the family history questionnaire. In the other pedigree, ambiguous information had been interpreted differently by the data administrator and genetic counsellor.

Family relationships The genetic counsellor pedigrees contained seven individuals where the data administrator had not been able to plot these individuals due to insufficient information about the family. This extra information was gained by the genetic counsellor in a phone call to confirm family details. There was variance in the position of four individuals between the genetic counsellor and data administrator pedigrees. In one of these cases, concerning remarriage, this was due to the data administrator's lack of experience in the use of Cyrillic. In the other three, the questionnaire information had been interpreted differently.

Clinical/medical information There was additional clinical/medical information on the genetic counsellor pedigrees for 11 individuals from nine pedigrees. For six individuals, information pertained to their cancer status. Three were marked by the genetic counsellor as affected, but plotted as unaffected by the data administrator. There was additional genetic counsellor information about cancer type for two individuals and about age of diagnosis for five. The source of the extra data was the referral letter in two of the cases, a telephone call from the genetic

Table 1 Differences, with reasons, between genetic counsellor (GC) and data administrator (DA) pedigrees

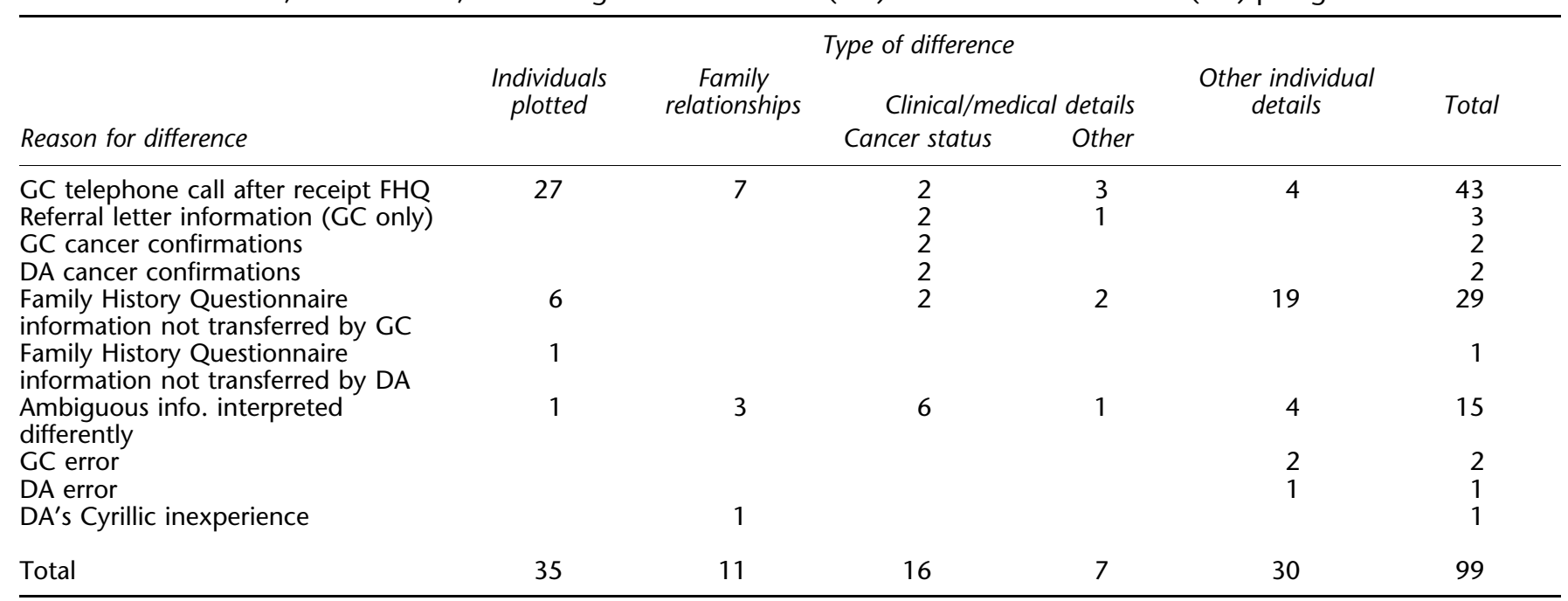


counsellor and from cancer confirmations. Information relating to separate medical issues (eg heart attack) was added to the pedigree for five individuals; from a telephone call in three cases, and a referral letter in one case.

The data administrator pedigrees contained additional clinical information for 12 individuals from seven families. The data administrator had marked seven individuals as affected (one cancer of the stomach, six cancer unknown), whereas the genetic counsellor had specified these cases as unaffected. This appeared to be due to questionnaire information not being transferred in one case and a differing interpretation of ambiguous questionnaire information in six others. The data administrator had transferred the age of diagnosis from the questionnaire for one individual (lung cancer), while two confirmations performed by the data administrator alone (the counsellors had not confirmed these cases) revealed the age of diagnosis for one individual (cancer of the nasal cavity) and an additional cancer type (colon cancer on a breast cancer pedigree) not cited on the questionnaire.

Other individual details Other differences between the pedigrees concerned the addition of extra information about family members such as name, dates of birth and death and gender. Additional information on the counsellor pedigrees was typically obtained from telephoning the proband to verify family history details after receipt of the questionnaire. The additional information on the data administrator pedigrees was due to transferring extra details from the family history questionnaires. Some differences (two of names, two of dates of birth) were due to differing interpretation of ambiguous questionnaire information and in two cases due to genetic counsellor error.

\section{Discussion}

A comprehensive and accurate family history is necessary for genetic risk assessment and appropriate management decisions. This study confirms that 'worked up' family pedigrees are indeed generated through complex processes. ${ }^{18}$ Constructing a pedigree from a family history questionnaire is not always a case of transferring clear-cut data. The information can be ambiguous and unclear, often due to the patient's lack of knowledge about their family history and sometimes due to poor handwriting. The data will require careful consideration of how it should be transposed, and on occasion it will be apparent that discussion with the proband is required. Once the initial pedigree is plotted, unless there is a blanket protocol of confirming all cancers, a decision then needs to be made about which cancers to confirm. A sound knowledge of the pedigree plotting software is a prerequisite to accurate representation of the data from the questionnaire and subsequent disease confirmations. At different stages careful consideration and evaluation of the data in hand is required and this study illustrates some of the competencies required at these points.

Delegating responsibility for disease confirmations does not appear to adversely compromise the quality of data obtained. In the cases where comparison was possible, information gathered by the data administrator mirrored that of the genetic counsellors in eight of nine cases (89\%). Genetic counsellors and data administrators collect data with different perspectives. Whereas, counsellors use data for making decisions, administrators are more concerned with establishing uniformity and perhaps obtaining more information than is directly relevant to clinical practice.

This study explored the implications of confirming all cancers to limit genetic counsellor involvement in early stages of pedigree generation. The data administrator's attempted confirmations of deceased cases not attempted by the genetic counsellors, constituted significant additional activity. While a blanket protocol of confirming all cancers before review by clinical staff may alleviate the pressure on genetic counsellors, this approach has its drawbacks. Firstly, there is a resource implication in having administrators dedicated to a significant amount of extra activity with arguably, limited clinical value. The additional information gained from confirmations by the data administrator alone (two cases) did not contribute significantly to the family histories in question. Furthermore, an important ethical consideration is that the blanket confirmation of all cancers can only apply to deceased individuals since there is no consent issue for access to medical records. It would be inappropriate to seek consent from living affected relatives if their risk status was not significant to the assessment and also to request other personnel to obtain unnecessary clinical information.

A notable difference between pedigrees was the number of pieces of information $(n=29)$ from the questionnaires not transferred by the genetic counsellors. These were primarily additional details, such as name, date of birth, that would not affect risk assessment, but nevertheless the inclusion of which may improve clinician knowledge of family details. This information was not transferred by the genetic counsellors due to time limitations; an advantage of a data administrator dedicated to pedigree activity is the ability to transfer all detail from the family history questionnaire.

An examination of differences between pedigrees highlighted the additional amount of information gained from sources other than the family history questionnaire. Notably, the genetic counsellors telephoning the patient after receipt of the questionnaire accounted for 43 extra pieces of information, primarily affecting the number of individuals plotted. Of all the reasons for differences, this was the most prolific and suggests that it is a prerequisite for maintaining quality and accuracy in any new protocol for family history data collection. 
The study suggests that again, clinical review will be necessary to determine when a phone call is necessary; the data administrator's perception of when it was necessary to call did not completely correspond with that of the genetic counsellor's. The data administrator perceived a telephone call necessary in twice as many cases as the counsellor actually made and in one case did not perceive contact to be necessary where the counsellor had called. It was not the remit of this study to explore whether a nonclinical administrator could make such phone calls, but it is apparent that sensitive interviewing skills are needed when discussing family history. The interviewer must also have the capacity to realise the relevance of certain information, such as primary versus secondary cancer, that ovarian cancer can be a secondary of breast cancer, but not viceversa and the significance of bilateral primary tumours. Such expertise feeds from tacit knowledge, largely acquired through clinical experience. It is arguable whether the competencies required for identifying the need for family history clarification and subsequent interviewing could be expected of a nonclinical data administrator. Schouten et $\mathrm{al}^{15}$ demonstrated in the Netherlands that registration personnel are able to collect cancer data with a high degree of accuracy and reliability. Brewster et al ${ }^{13}$ argue cancer registries are increasingly becoming involved in supporting the counselling activities of genetic services.

We acknowledge that the redirection of all pedigree activity to nonclinical personnel up to the point of risk assessment is not possible. The soundness of medical decisions can be compromised if the history on affected relatives is inaccurate. ${ }^{10}$ Therefore, there is a need for review by clinical staff after the initial plotting of the pedigree to determine which cancers to confirm; whether to contact patients to clarify family history information; and to give the data administrator an opportunity to discuss ambiguous details on the family history questionnaire. Once these issues have been resolved the data administrator can confirm disease cases on the clinical databases available and request confirmations from other hospitals and cancer registries. The subsequent data can be amended to the pedigrees and passed to the clinicians for risk review.

This pilot study contains modest but significant conclusions for the construction of pedigrees. There was a good degree of concordance between pedigrees constructed by a nonclinical data administrator and those of experienced genetic counsellors. While the data administrator for this study (a humanities graduate) was somewhat unusual, further research with NHS administrative staff - and within different models of service delivery - will be necessary. This study demonstrates that role substitution for pedigree construction and disease confirmation is feasible; however, future research needs to explore more thoroughly the effects on work patterns of all staff in cancer genetics clinics; the health economic implications of the time spent by each professional to obtain the data; the economic impact of using electronic versus labour-intensive methods of data collection and collation, and finally how such changes would be perceived by other agencies as well as patients and their families. We also need to think more clearly about who should have access to cancer data, in what form and in ways that do not contravene data protection legislation in Europe.

\section{Acknowledgements}

Vanessa Tempest, Rachel Iredale and Jonathon Gray are supported by Tenovus, the cancer charity. We thank Joyce Cooper, June Williams and Stephanie Sivell.

\section{References}

1 Leeson S, Iredale R, Stansfield K, Evans A, Gray J: Cancer genetics in Wales: opinions of gynaecologists on the management of individuals at risk of familial ovarian cancer. Eur J Cancer Care 2001; 10: 172-179.

2 Wonderling D, Hopwood P, Cull A et al: A descriptive study of UK cancer genetics services: an emerging clinical response to the new genetics. Br J Cancer 2001; 85: 166-170.

3 Steel M, Smyth E, Vasen $\mathrm{H}$ et al: Ethical, social and economic issues in familial breast cancer: a compilation of views from the EC Biomed II Demonstration Project. Dis Markers 1999; 15: $125-131$.

4 Lucassen A, Watson E, Harcourt J, Rose P, O'Grady J: Guidelines for referral to a regional genetics service: GPs respond by referring more appropriate case. Fam Pract 2001; 18: 135-140.

5 Gray J, Brain K, Iredale R, Alderman J, France E, Hughes H: A pilot study of telegenetics. J Telemed Telecare 2000; 6: 245-247.

6 Department of Health: The NHS Cancer Plan, a Plan for Investment, a Plan for Reform. London: Department of Health, 2000.

7 Brain K, Norman P, Gray J, Rogers C, Mansel R, Harper P: A randomized trial of specialist genetic assessment: psychological impact on women at different levels of familial breast cancer risk. Br J Cancer 2002; 86: 233-238.

8 Eerola H, Blomqvist C, Pukkala E, Pyrhonen S, Nevanlinna H: Familial breast cancer in southern Finland: how prevalent are cancer families and can we trust the family history reported by patients? Eur J Cancer 2000; 36: 1143-1148.

9 Murff HJ, Spigel DR, Syngal S: Does this patient have a family history of cancer? An evidence based analysis of the accuracy of family cancer history. JAMA 2004; 292: 1480-1489.

10 Sijmons RH, Boonstra AE, Reefhuis $\mathrm{J}$ et al: Accuracy of family history of cancer: clinical genetics implications. Eur J Hum Genet 2000; 8: 181-186.

11 Ziogas A, Anton-Culver H: Validation of family history data in cancer family registries. Am J Prev Med 2003; 24: 190-198.

12 Douglas FS, O'Dair LC, Robinson M, Evans DGR, Lynch SA: The accuracy of diagnoses as reported in families with cancer: a retrospective study. J Med Genet 1999; 36: 309-312.

13 Brewster DH, Fordyce A, Black RJ: Impact of a cancer registrybased genealogy service to support clinical genetics services. Fam Cancer 2004; 3: 139-141.

14 Ivanovich J, Babb S, Goodfellow P et al: Evaluation of the family history collection process and the accuracy of reporting among a series of women with endometrial cancer. Clin Cancer Res 2002; 8: $1849-1856$.

15 Schneider KA, DiGianni LM, Patenaude AF et al: Accuracy of cancer family histories: comparison of two breast cancer syndromes. Genet Test 2004; 8: 222-228. 
16 Schouten LJ, Jager JJ, van den Brandt PA: Quality of cancer registry data: a comparison of data provided by clinicians with those of registration personnel. Br J Cancer 1993; 68: 974-977.

17 Simonato L, Zambon P, Rodella S et al: A computerised cancer registration network in the Veneto region, north-east of Italy. $\mathrm{Br} \mathrm{J}$ Cancer 1996; 73: 1436-1439.

18 Bennett RL, Steinhaus KA, Uhrich SB, O'Sullivan C: The need for developing standardized family pedigree nomenclature. J Genet Couns 1993; 2: 261-273.

19 Wood F, Prior L, Gray J: Translations of risk: decision making within a cancer genetics clinic. Health Risk Soc 2003; 5: 185-198.
20 Calman K, Hine D: A Policy Framework for Commissioning Cancer Services. London: Department of Health, 1995.

21 Harper P: Genetics and Cancer Services. London: Department of Health, 1998.

22 Claus EB, Schildkraut JM, Thompson WD, Risch NJ: The genetic attributable risk of breast and ovarian cancer. Cancer 1996; 77 : $2318-2324$

23 Hodson S, Milner B, Brown I et al: Clinical service development for familial breast cancer in European countries: the role and training of genetic counsellors in this service, and educational provision in cancer genetics. J Gynaecol Oncol 2000; 5: 261-268. 